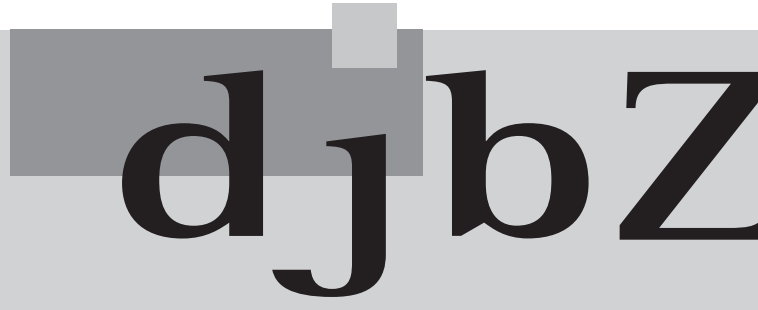

Zeitschrift des Deutschen Juristinnenbundes

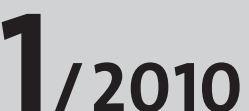

13. Jahrgang, Seite 1-46

Herausgeber: Deutscher Juristinnenbund e. V.

Präsidium: Jutta Wagner, Rechtsanwältin und Notarin, Fachanwältin für Familienrecht, Berlin (Präsidentin); Renate Maltry, Rechtsanwältin, Fachanwältin für Familienrecht und Erbrecht, München; Ramona Pisal, Vorsitzende Richterin am Oberlandesgericht, Brandenburg a.d.H. (Vizepräsidentinnen); Dagmar Brinkmann, Regierungsdirektorin, Frankfurt a.M. (Schatzmeisterin); Schriftleitung: Anke Gimbal, Rechtsassessorin, Geschäftsführerin Deutscher Juristinnenbund, Berlin.

\title{
Die Praxis der Anerkennung von ausländischen Abschlüssen in der Diskussion
}

\section{Dr. Bettina Englmann \\ gGmbH „Tür an Tür Integrationsprojekte“, Augsburg’}

Akteur(inn)e(n) des Bundes und der Länder diskutieren seit der Vorlage des Nationalen Integrationsplans (2007) über die Anerkennung von ausländischen Qualifikationen. Zuwander(innen)er sind am deutschen Arbeitsmarkt von hoher Arbeitslosigkeit und Dequalifizierung betroffen; es stellt sich die Frage, wie ihr Zugang zum Arbeitsmarkt verbessert werden kann. Das Bundesamt für Migration und Flüchtlinge verpflichtete sich, Handlungsvorschläge zur beruflichen Anerkennung zugewanderter Akademiker(innen) im Rahmen des bundesweiten Integrationsprogramms zu erarbeiten; zudem wurde ein zielgruppenspezifisches Konzept für Ärzt(inn)e(n) angekündigt. Eine Veröffentlichung beziehungsweise Umsetzung des Programms ist mittlerweile für den Herbst 2009 angekündigt. Im Beitrag der Länder wurde ebenfalls auf die wichtige Rolle der Anerkennung von ausländischen Abschlüssen hingewiesen, allerdings ohne Einschränkung auf bestimmte Berufsgruppen: „Die Länder halten es für erforderlich, dass die von den Zugewanderten im Ausland erworbenen Schul-, Bildungs- und Berufsabschlüsse volkswirtschaftlich besser genutzt werden. Dies kann ggf. auch Teilanerkennungen und gezielte Nachqualifizierungen einschließen.“2

Bisher ist der Zugang zu einem Anerkennungsverfahren nicht für jede(n) Inhaber(in) einer ausländischen Qualifikation vorgesehen. In Deutschland hängen Anerkennungsmöglichkeiten von der Zugehörigkeit zu einer bestimmten Migrantengruppe, vom Beruf und vom Bundesland $a b$, in dem der (die) potenzielle Antragsteller(in) wohnt. ${ }^{3}$ Im Rahmen der Bildungshoheit sind die Bundesländer für die Durchführung von Anerkennungsverfahren zuständig. Nur Spätaussiedler(innen) haben durch Bestimmungen des Bundesvertriebenengesetzes das Recht, für jeden Berufsabschluss eine Anerkennung und damit Gleichstellung mit einem vergleichbaren deutschen zu beantragen. Im Bereich der reglementierten Berufe sind EUBürger(innen) durch die Regelungen der EU-Anerkennungsrichtlinien privilegiert, welche unter anderem das System der Teilanerkennung eingeführt haben. Antragsteller(innen) können in diesem Fall einen Ausgleichsmechanismus nutzen, der wahlweise in einer Eignungsprüfung, die sich nur auf festgestellte individuelle Defizite beziehen darf, oder einer Anpassungsmaßnahme besteht. Nach Abschluss des Verfahrens erfolgt eine volle Anerkennung und damit Formalisierung der ausländischen Qualifikation. Bisher profitieren vor allem EUBürger(innen) von diesem Angebot: In Gesundheitsfachberufen führen Fachschulen Anpassungsqualifizierungen durch, bei Erzieher(inne)n ist die Auflage eines Praktikums verbreitet, das oft mit einem Fachgespräch abgeschlossen wird. Inhaber(innen) von Drittlandsdiplomen haben in der Regel keinen Anspruch auf die Verknüpfung von Anerkennung und Anpassungsqualifizierung, sodass sich Teilanerkennungen in der Praxis als Nicht-Anerkennungen auswirken.

Wenn Migrant(inn)en mit einschlägigen Qualifikationen nicht einmal Zugang zu einem Anerkennungsverfahren haben,

1 Im Rahmen des Netzwerks „Integration durch Qualifizierung“ führte Dr. Englmann zusammen mit Dr. Martina Müller eine wissenschaftliche Untersuchung der deutschen Anerkennungspraxis durch, deren Ergebnisse in der Studie „Brain Waste“ (2007) vorliegen. Um das Wissen über Anerkennungsmöglichkeiten und zuständige Stellen der Länder für eine breite Öffentlichkeit zugänglich zu machen, richteten sie 2008 das Informationsportal www.berufliche-anerkennung.de ein.

2 Die Bundesregierung, Der Nationale Integrationsplan. Neue Wege - Neue Chancen, Berlin 2007, 28.

3 Zur deutschen Anerkennungspraxis vgl. Englmann, Bettina/Müller, Martina, Brain Waste. Die Anerkennung von ausländischen Qualifikationen in Deutschland, Augsburg 2007. 
dann wird eine erfolgreiche Integration trotz vorliegender Fachkompetenz behindert. Dies kann zu sozialer Exklusion führen. In diesem Kontext spielt auch eine Rolle, „wie sehr sich die Zuwanderer integriert, akzeptiert und dieser Gesellschaft zugehörig fühlen“. Durch die Allensbach-Befragung von Zuwander(innen)ern im Frühjahr 2009 wurde deutlich, dass die individuell empfundene soziale Anerkennung von Seiten der Gesellschaft als nicht ausreichend gesehen wird. Gefragt wurde unter anderem „nach der Art der persönlich erlebten Diskriminierung “ am Arbeitsmarkt, eine Antwort war, dass „gleichwertige Bildungsabschlüsse, die nicht in Deutschland erworben wurden, (...) nicht anerkannt" werden. ${ }^{4}$ Die jahrelangen Versäumnisse bei der Integration von Migrant(inn) en wirken sich nicht nur für die Einzelnen negativ aus. Der sich abzeichnende demografische Wandel stellt die Volkswirtschaften Europas vor die Herausforderung, qualifizierte Zuwander(innen)er anzuwerben. Mit der Verknappung der Arbeitskräfte steigt auch der Druck, die inländischen Qualifikationsreserven besser auszuschöpfen.

Anlässlich des gemeinsamen Bildungsgipfels im Oktober 2008 einigten sich die Bundesregierung und die Regierungschefs der Länder auf eine umfassende Qualifizierungsinitiative. Ein besonderer Schwerpunkt der angestrebten Reformen liegt auf der Teilhabe der Einzelnen an den Bildungsangeboten und damit auf der Bedeutung sozialer Inklusion. Ein Aufstieg durch Bildung soll gleichermaßen für die Bevölkerung mit Migrationshintergrund möglich sein. Neue Chancen könnten sich für Zuwander(innen)er eröffnen, die Qualifikationen aus ihren Herkunftsländern mitbringen: „Bis Mitte 2009 werden Bund und Länder entscheiden, inwieweit bestehende Anerkennungsverfahren [...] ausgeweitet werden können. Im Ausland erworbene Abschlüsse sollen zügig auf Anerkennung geprüft und ggf. auch Teilanerkennungen ausgesprochen werden. Der Bund unterstützt bei Teilanerkennungen mit geeigneten Förderungen von Ergänzungs- und Anpassungsqualifizierungen. " ${ }^{5}$ Ein wichtiger Schritt, der auf den Bildungsgipfel folgte, war die Einrichtung einer Bund-Länder-Arbeitsgruppe, die Vorschläge für eine Verbesserung der Anerkennungspraxis in Deutschland erarbeiten wird. Erste Ergebnisse sind im Herbst 2009 zu erwarten. Dabei kann auf diverse Vorarbeiten zurückgegriffen werden, die zuletzt in Bund, Ländern und Kommunen diskutiert wurden.

\section{Bemühungen um strukturelle Änderungen in den Bundes- ländern}

Ein Ziel des Nationalen Integrationsplans war ein Perspektivwechsel im Blick auf Personen mit Migrationshintergrund. Ihre Potenziale für Wirtschaft und Gesellschaft sollten stärker gewürdigt werden. Die festgelegten Selbstverpflichtungen umfassen Institutionen des Bundes, der Länder, der Kommunen, die Sozialpartner und zahlreiche weitere Akteure.

Auch auf Länderebene wurden Integrationspläne vorgelegt, die sich in Umfang und strategischen Absichten unterscheiden. Im Kontext einer besseren Platzierung von $\mathrm{Mi}$ grant(inn)en am Arbeitsmarkt erfolgen in der Regel auch
Erwähnungen der Anerkennungspraxis von ausländischen Abschlüssen. In Baden-Württemberg legte Justizminister Goll, der auch Integrationsbeauftragter der Landesregierung ist, im September 2008 einen Landesintegrationsplan vor. Er kündigte an zu prüfen, „ob und inwiefern eine Vereinfachung des Verfahrens zur Anerkennung von ausländischen Abschlüssen (...) möglich ist" ". ${ }^{6}$ Wie eine Vereinfachung gestaltet werden könnte, wurde nicht erläutert.

Im Integrationskonzept des Landes Rheinland-Pfalz (Juli 2007) wurde ebenfalls eine Vereinfachung und Erleichterung der Anerkennung thematisiert. Außerdem wurde die konkrete Absicht geäußert, bestehende Hürden für Drittstaatsangehörige zu erfassen und abzubauen. ${ }^{7}$ Eine ähnliche Formulierung findet sich im „Ersten Umsetzungsbericht zum Berliner Integrationskonzept 2007-2009“: „In der Anerkennung von im Herkunftsland erworbenen Qualifikationen liegt eine große arbeitsmarktpolitische und integrationspolitische Chance. Anerkennungsprozesse müssen jedoch noch weiter entbürokratisiert und vereinfacht werden, damit Fachkräfte aus dem Ausland in Deutschland leichter Arbeit finden." 8

Auch Länderparlamente beschäftigten sich mit der Anerkennungspraxis. Die Bremische Bürgerschaft forderte den Senat im Oktober 2008 auf, Maßnahmen zur „Vereinheitlichung der Anerkennungsverfahren unter Einbeziehung der EU-Richtlinien “ zu ergreifen. ${ }^{9}$ Diese weitreichende Forderung zielt auf eine Umsetzung der Anerkennungsrichtlinie 2005/36/ EG für Inhaber(inne)n von Drittlandsdiplomen. Bisher werden in deutschen Berufsqualifikationsgesetzen für reglementierte Berufe fast ausschließlich EU-Bürger(innen) bezüglich einer Würdigung von Berufserfahrung sowie der Möglichkeit der Teilanerkennung privilegiert. In einem ersten Zwischenbericht stellte der Senat die Verwaltungspraxis Bremens zwar umfassend dar, eine Ausweitung der bestehenden Rechtsansprüche wurde jedoch nicht erwähnt.

Auch in Schleswig-Holstein legte die Landesregierung 2009 einen vergleichbaren Bericht vor. In seinen abschließenden „Empfehlungen zur Verbesserung der schleswig-holsteinischen Anerkennungspraxis“ wird ein Problem sichtbar, das sich ge-

4 Bertelsmann Stiftung (Hrsg.), Zuwanderer in Deutschland. Ergebnisse einer repräsentativen Befragung von Menschen mit Migrationshintergrund. Durchgeführt durch das Institut für Demoskopie Allensbach, Gütersloh 2009, 46 und $70 \mathrm{f}$.

5 Die Bundesregierung/Die Regierungschefs der Länder, Aufstieg durch Bildung. Die Qualifizierungsinitiative für Deutschland, Dresden, 22. Oktober 2008, $11 \mathrm{f}$.

6 Der Integrationsbeauftragte der Landesregierung Baden-Württemberg, Integration gemeinsam schaffen. Integrationsplan BadenWürttemberg, Stuttgart 2008, 74.

7 Ministerium für Arbeit, Soziales, Gesundheit, Familie und Frauen Rheinland-Pfalz, Verschiedene Kulturen - Leben gemeinsam gestalten! Integrationskonzept des Landes Rheinland-Pfalz, Mainz 2007, 19.

8 Senatsverwaltung für Integration, Arbeit und Soziales, Der Beauftragte des Senats von Berlin für Integration und Migration, Erster Umsetzungsbericht zum Berliner Integrationskonzept 2007-2009, Berlin 2009, 29.

9 Bremische Bürgerschaft, Mitteilung des Senats vom 12. Mai 2009, Anerkennung von im Ausland erworbenen Abschlüssen - Ein Beitrag zur Bekämpfung des Fachkräftemangels, Drs. 17/779, 1. 
nerell in den Ländern zeigt: Eigene Initiativen auf Landesebene, die sich aufgrund der Zuständigkeit anbieten, werden derzeit nur zögerlich erwogen. „Konkrete Empfehlungen zur Verbesserung der schleswig-holsteinischen Anerkennungspraxis können nur vor dem Hintergrund betrachtet werden, dass es keine länderspezifische Praxis geben sollte. Die Verfahren sind auf Bundes- und EU-Ebene abzustimmen. Deshalb gilt es derzeit abzuwarten, wie die Selbstverpflichtungen des Bundes aus dem Nationalen Integrationsplan umgesetzt werden."10 Die rechtlichen und verwaltungspraktischen Unterschiede der Anerkennung sind in den Ländern jedoch längst vorhanden; um sie zu beseitigen, werden Aktivitäten des Bundes oder gar der Europäischen Union kaum ausreichen. Bisher fehlen praktische Lösungsansätze in den Ländern, um eine Verbesserung der beruflichen Anerkennung zu gestalten, so dass sie positive Auswirkungen auf die Arbeitsmarkterfolge von Migrant(inn)en haben könnte. Es verwundert daher kaum, dass die Selbstverpflichtungen der Länder hier keine Erwähnung fanden. Im „Ersten Fortschrittsbericht zum Nationalen Integrationsplan“ vom Herbst 2008 findet sich zwar ein Abschnitt „Anerkennung ausländischer Abschlüsse“ im Kontext der „Umsetzung des Länderbeitrags“, doch dieser Abschnitt ist bemerkenswert frei von zukünftigen Handlungsabsichten oder gar einer Umsetzung; stattdessen finden sich hier Hinweise auf die bestehende gute Praxis einzelner Kammern und Anpassungsqualifizierungsprojekte sowie der Zentralstelle für ausländisches Bildungswesen. ${ }^{11}$

\section{Das Thema Anerkennung im Bund}

Umso aktionsfreudiger zeigten sich in den vergangenen Monaten Institutionen des Bundes. Insbesondere die Integrationsbeauftragte der Bundesregierung, das Bundesministerium für Arbeit und Soziales und das Bundesministerium für Bildung und Forschung brachten innovative Vorschläge für eine Reform der beruflichen Anerkennung ein. Im Juni 2009 wurden zwei Eckpunktepapiere vorgelegt, die unter anderem eine Ausweitung der Rechtsansprüche für Inhaber(innen) ausländischer Abschlüsse vorsehen. Zudem soll das Angebot individueller Kompetenzfeststellungen ausgebaut werden. ${ }^{12}$

Das Engagement des Bundes wird ebenfalls von parlamentarischen Initiativen flankiert. Der Deutsche Bundestag debattierte am 29. Januar 2009 Chancen und Probleme der deutschen Anerkennungspraxis. ${ }^{13}$ Im Vorfeld hatten zweiten Oppositionsparteien Anträge zum Thema gestellt. Die Fraktion der Linken warb „Für eine erleichterte Anerkennung von im Ausland erworbenen Schul-, Bildungs- und Berufsabschlüssen“. Die Fraktion der FDP schlug unter dem Titel „Lebensleistung von Migrantinnen und Migranten würdigen - Anerkennungsverfahren von Bildungsabschlüssen verbessern“ einen grundsätzlichen Paradigmenwechsel hin zu einer aktiven Willkommenskultur vor. Sie forderte unter anderem einen gesetzlichen Anspruch auf ein Anerkennungsverfahren für alle aufenthaltsberechtigten Inhaber(innen) ausländischer Qualifikationen. Redner(innen) aller Parteien betonten in der Debatte den politischen Handlungsdruck, da eine erfolgreiche beruf- liche Integration von Migrant(inn)en im Hinblick auf den demografischen Wandel eine immer wichtigere Rolle spielen wird.

Vor diesem Hintergrund ist eine umfassende Reform der beruflichen Anerkennung im Bund in greifbare Nähe gerückt.

\section{Reformen durch Rechtsprechung?}

Doch sogar wenn die zahlreichen Absichtserklärungen und Prüfaufträge in Bund und Ländern nicht in absehbarer Zeit zu Reformen der Anerkennungspraxis führen, könnte neuer Handlungsdruck durch Urteile der Verwaltungsgerichte entstehen. In den vergangenen Jahren hatten im Feld der beruflichen Anerkennung insbesondere Urteile des Europäischen Gerichtshofes große Auswirkungen: Bei Lehrer(inne)n und Rechtsanwält(inn)en haben erfolgreiche Klagen dazu geführt, dass zumindest EU-Bürger(inne)n eine Anpassungsmaßnahme beziehungsweise eine Eignungsprüfung angeboten werden muss. Zuvor waren Anerkennungsanträge mit dem Hinweis auf wesentliche Unterschiede vielfach abgelehnt worden, ohne den Ausgleichsmechanismus der Teilanerkennung anzuwenden, den die EU-Anerkennungsrichtlinien vorsehen.

Seit Dezember 2008 liegt ein Urteil des Bundesverwaltungsgerichts vor, das ebenfalls große Auswirkungen auf die Praxis der Anerkennungsstellen entfalten könnte. ${ }^{14}$ Erfolgreich geklagt hatte eine in Russland ausgebildete Ärztin. Sie verlangte die Erteilung der Approbation auf der Grundlage eines gleichwertigen Ausbildungsstandes. Im Urteil wurde ausdrücklich betont, dass neben einem objektiven Vergleich der Ausbildungsgänge auch die individuell erworbene Qualifikation sowie die Berufserfahrung im Herkunftsland und in Deutschland zu berücksichtigen seien, um die Gleichwertigkeit zu prüfen.

Bislang sah die Praxis der Approbationsstellen vor, dass eine Approbation in den meisten Fällen das Bestehen einer Kenntnisstandprüfung voraussetzte. Ausnahmen bestanden für durch EU-Recht Privilegierte und für Angehörige weniger Drittstaaten wie USA oder Südafrika. Damit lag die höchst eigenartige Situation vor, dass ausländische Ärzt(inn)e(n) über Jahre hinweg mit einer Berufserlaubnis praktizierten, sogar den deutschen Facharzt mittels Facharztprüfung erwarben, aber immer noch eine Gleichwertigkeit ihrer Ausbildung mit-

10 Schleswig-Holsteinischer Landtag, Bericht der Landesregierung Anerkennung von im Ausland erworbenen Abschlüssen, Drs. 16/2525, 9 .

11 Deutscher Bundestag, Erster Fortschrittsbericht zum Nationalen Integrationsplan, Drs. 16/10800, 6.11.2008, $143 \mathrm{ff}$.

12 Bundesministerium für Arbeit und Soziales, Kompetenzen wahrnehmen, anerkennen und fördern. Vorschläge des BMAS für ein Gesetz zur Anerkennung ausländischer Qualifikationen, Berlin 28. Mai 2009. Und: Die Beauftragte der Bundesregierung für Migration, Flüchtlinge und Integration/Bundesministerium für Bildung und Forschung/Bundesministerium für Wirtschaft und Technologie/ Bundesministerium des Innern, Verbesserung der Feststellung und Anerkennung im Ausland erworbener beruflicher Qualifikationen und Abschlüsse. Eckpunkte, Berlin 18.6.2009.

13 Deutscher Bundestag, Plenarprotokoll 16/202, Berlin, Donnerstag, den 29. Januar 2009, 21872-21879.

14 BVerwG 3. Senat, 11.12.2008, 3 C 33/07. 
tels Kenntnisstandprüfung nachweisen mussten, wenn sie nach dem Erhalt der Staatsbürgerschaft eine Approbation beantragten. Dass die Klägerin diese Prüfung zweimal nicht bestanden hatte, wurde als nicht relevant erachtet. Auch der Hinweis, dass die Zentralstelle für ausländisches Bildungswesen, die als Gutachterstelle für Anerkennungsstellen wirkt, die russische Ausbildung nicht als objektiv gleichwertig ansieht und regelmäßig auf kürzere Schul- und Praktikazeiten verweist, wurde zurückgewiesen, da die über zwanzigjährige Berufserfahrung keine Rolle bei der Bewertung gespielt hatte. Der individuelle Kenntnisstand sei grundsätzlich zu prüfen, daher wurde auch dem Einwand bezüglich genereller bisheriger Erfahrungen der Anerkennungspraxis keine Bedeutung zugemessen, da er nichts über den jeweiligen Einzelfall aussage.

Obwohl das Gericht keine Diskriminierung der Klägerin in der Ungleichbehandlung gegenüber baltischen Staatsangehörigen sah, die eine automatische Anerkennung ihrer sowjetischen Arztausbildungen erlangen können, wurde doch auf spezifische Auswirkungen der Richtlinie 2005/36/EG hingewiesen. Demnach habe der deutsche Gesetzgeber keine Gefahr für die Bevölkerung erkennen können, als er einer Anerkennung baltischer Staatsangehöriger zustimmte; demnach sei ein gleichwertiger Kenntnisstand mit sowjetischen Ausbildungen durchaus möglich.

Es bleibt abzuwarten, welche Auswirkungen dieses Urteil für die deutsche Anerkennungspraxis haben wird. Dass ausländischen Ärzt(inn)en nach Erhalt der Staatsangehörigkeit nun eine Bewertung ihrer Berufserfahrung nicht mehr verweigert werden kann, um die Gleichwertigkeit festzustellen, könnte sich auch auf andere Berufe auswirken.

Kompetenzbasierte Anerkennungsverfahren als neuen Standard einzuführen, würde sich nicht nur integrationspolitisch positiv auswirken. Auch bildungspolitisch ist die Wertschätzung individuell feststellbarer Kompetenzen gegenüber der formalen Qualifikation, die vielleicht schon Jahre zurückliegt und nichts über tatsächlich vorliegende Kenntnisse und Fähigkeiten aussagt, im Fokus der europäischen Diskussionen um Modernisierungen im Bildungssystem. Der Europäische Qualifikationsrahmen, der derzeit erstellt wird, könnte auch im Bereich der Anerkennung von ausländischen Abschlüssen zu weiteren Reformen führen.

\section{Arbeitsmarktzugang und Ausbildungsbeihilfen}

\author{
Prof. Dr. Dorothee Frings \\ Hochschule Niederrhein, Mönchengladbach
}

\section{Patriarchalische Struktur des Ausländerrechts}

Migration wird traditionell als männliches Phänomen wahrgenommen - so bleibt auch das deutsche Ausländerrecht weiterhin auf den männlichen Arbeitnehmer fokussiert.

Wenn das Bild der migrantischen Frau in unserer Gesellschaft noch immer geprägt ist vom Stereotyp der Kopftuch tragenden muslimischen Hausfrau oder der fast sprachlosen Putzfrau, die abends ihren Putzwagen durch leere Büroflure schiebt, so liegt die Verantwortung nicht allein bei psychologisch begründeten Zuschreibungen und Medienbildern, sondern auch bei einer Ausländerpolitik, die Frauen als Anhängsel ihrer Ehemänner behandelt hat.

- Das Aufenthaltsrecht knüpft an der Sicherung des Lebensunterhalts und damit an der Erwerbstätigkeit an; andere gesellschaftliche Beiträge - wie die Kindererziehung und Pflege von Angehörigen - werden ausgeblendet. Sie können bis heute allenfalls als Härtegesichtspunkte in besonders gelagerten Ausnahmefällen berücksichtigt werden; in der Regel nur, wenn es zur Vermeidung von Grundrechtsbeeinträchtigungen unabweisbar ist.

- Bis 2004 wurde nachziehenden Ehefrauen in den entscheidenden ersten Jahren ihres Aufenthalts der Zugang zum Arbeitsmarkt versperrt und ihnen so gesetzlich die Hausfrauenrolle verordnet.
- Familienbezogene Sozialleistungen galten bis vor wenigen Jahren als öffentliche Leistungen, die der Erteilung und Verlängerung der Aufenthaltserlaubnis entgegenstanden. Bis heute wird das Wohngeld als eine aufenthaltsschädliche Sozialleistung behandelt. Kinder zu haben wird immer noch zum Risiko des Aufenthaltsverlustes.

- Durch die Nichtanerkennung der ausländischen Qualifikationen werden Migrantinnen als „naturbegabt“ für die am schlechtesten bezahlten Bereiche personaler Dienstleistungen bewertet und in den prekären Sektor des Arbeitsmarktes abgedrängt.

Der erste Ausländerbeauftrage der Bundesregierung, Heinz Kühn, kritisierte in seinem berühmten Memorandum bereits vor genau 30 Jahren die Vernachlässigung der Arbeitsmarktintegration von Migrantinnen: „Mit gleicher Intensität müssen Möglichkeiten zur berufsbegleitenden Höherqualifizierung der ungelernten Beschäftigung, insbesondere auch der vielfach besonders benachteiligten ausländischen Mädchen ausgeschöpft werden. “1

Kühn kritisierte auch die Arbeitsmarktprüfung, nach der die Arbeitserlaubnis nur erteilt werden darf, wenn für den konkreten Arbeitsplatz keine sonstigen Arbeitsuchenden ver-

1 Kühn, Heinz, Stand und Weiterentwicklung der Integration der ausländischen Arbeitnehmer und ihrer Familien in der Bundesrepublik Deutschland, 6.5, Kühn-Memorandum, September 1979, erhältlich im Internet: <http://www.migration-online.de/data/ khnmemorandum_1.pdf $>$ (besucht am 12. Oktober 2009). 